\title{
Neutrino Mixing as a Source for Cosmological Constant
}

\author{
M. Blasone ${ }^{b \sharp}$, A. Capolupo ${ }^{b}$, S. Capozziello ${ }^{b}$, S. Carloni ${ }^{\natural}$, and G. Vitiello ${ }^{b \sharp}$ \\ bDipartimento di Fisica “E.R. Caianiello” and INFN, Università di Salerno, I-84100 Salerno, Italy \\ \# Unità INFM, Salerno, Italy \\ ${ }^{\natural}$ Department of Applied Mathematics, University of Cape Town, South Africa
}

Received on 19 December, 2004

\begin{abstract}
We report on recent results showing that neutrino mixing may lead to a non-zero contribution to the cosmological constant. This contribution is of a completely different nature with respect to the usual one by a massive spinor field. We also study the problem of field mixing in Quantum Field Theory in curved space-time, for the case of a scalar field in the Friedmann-Robertson-Walker metric.
\end{abstract}

\section{Introduction}

The problem of cosmological constant is currently one of the most challenging open issues in theoretical physics and cosmology. The main difficulty comes from the fact that, when estimating its value as a gravitational effect of vacuum energy, the numerical results are in strong disagreement with the accepted upper bound $\Lambda<10^{-56} \mathrm{~cm}^{-2}$ [1].

We show here that a new contribution to the vacuum energy and therefore to the cosmological constant may arise from neutrino mixing [2]. The contribution we find comes from the specific nature of the field mixing and is therefore of completely different origin with respect to the ordinary vacuum energy contribution of a massive spinor field.

Indeed, it has been shown [3]-[13] that the vacuum for fields with definite masses is not invariant under the field mixing transformations and in the infinite volume limit, it is unitarily inequivalent to the vacuum for the fields with definite flavor. In the case of neutrinos, this results in a condensate of neutrino-antineutrino pairs, with a density related to the mixing angle(s) and mass difference(s) among the different generations. Phenomenological consequences of such a nontrivial condensate structure of the flavor vacuum have been studied for neutrino oscillations [8] and for beta decay [9].

In this paper we consider the case of two flavors and Dirac neutrino fields, although the conclusions we reach can be easily extended to the case of three flavors and Majorana neutrinos $[11,12]$.

We also include a preliminary study of mixed (bosonic) fields in a curved background, for the case of FRW metric.

In Section 2, we shortly summarize the main results for neutrino mixing in QFT. In Section 3, we compute the neutrino contribution to the cosmological constant and estimate its value by using the natural scale of neutrino mixing as a cut-off. The result turns out to be compatible with the currently accepted upper bound on $\Lambda$. In Section 4, quantum fields and mixing relations are analyzed in expanding universe. Section 5 is devoted to conclusions.

\section{Neutrino mixing in Quantum Field Theory}

The main features of the QFT formalism for the neutrino mixing are summarized below. For a detailed review see [13]. For sake of simplicity, we consider the two flavor case and we use Dirac neutrino fields. The Lagrangian density describing the Dirac neutrino fields with a mixed mass term is:

$$
\mathcal{L}(x)=\bar{\Psi}_{f}(x)(i \not \partial-M) \Psi_{f}(x),
$$

where $\Psi_{f}^{T}=\left(\nu_{e}, \nu_{\mu}\right)$ and $M=\left(\begin{array}{cc}m_{e} & m_{e \mu} \\ m_{e \mu} & m_{\mu}\end{array}\right)$. The relation between Dirac fields $\Psi_{f}(x)$, eigenstates of flavor, and Dirac fields $\Psi_{m}(x)$, eigenstates of mass, is given by

$$
\Psi_{f}(x)=\mathcal{U} \Psi_{m}(x),
$$

with $\Psi_{m}^{T}=\left(\nu_{1}, \nu_{2}\right) \cdot \mathcal{U}$ is the mixing matrix

$$
\mathcal{U}=\left(\begin{array}{cc}
\cos \theta & \sin \theta \\
-\sin \theta & \cos \theta
\end{array}\right)
$$

being $\theta$ the mixing angle. Using Eq.(3), we diagonalize the quadratic form Eq.(1), which then reduces to the Lagrangian for the Dirac fields $\Psi_{m}(x)$, with masses $m_{i}, i=1,2$ :

$$
\mathcal{L}(x)=\bar{\Psi}_{m}(x)\left(i \not \partial-M_{d}\right) \Psi_{m}(x),
$$

where $M_{d}=\operatorname{diag}\left(m_{1}, m_{2}\right)$. The mixing transformation (2) can be written as [3]

$$
\nu_{\sigma}(x) \equiv G_{\theta}^{-1}(t) \nu_{i}(x) G_{\theta}(t),
$$

where $(\sigma, i)=(e, 1),(\mu, 2)$, and the generator $G_{\theta}(t)$ is given by

$$
G_{\theta}(t)=\exp \left[\theta \int d^{3} \mathbf{x}\left(\nu_{1}^{\dagger}(x) \nu_{2}(x)-\nu_{2}^{\dagger}(x) \nu_{1}(x)\right)\right] .
$$


The free fields $\nu_{i}(\mathrm{i}=1,2)$ are given, in the usual way, in terms of creation and annihilation operators (we use $t \equiv x_{0}$ ):

$$
\nu_{i}(x)=\sum_{r} \int \frac{d^{3} \mathbf{k}}{(2 \pi)^{\frac{3}{2}}}\left[u_{\mathbf{k}, i}^{r}(t) \alpha_{\mathbf{k}, i}^{r}+v_{-\mathbf{k}, i}^{r}(t) \beta_{-\mathbf{k}, i}^{r \dagger}\right] e^{i \mathbf{k} \cdot \mathbf{x}},
$$

with $i=1,2$,

$$
u_{\mathbf{k}, i}^{r}(t)=e^{-i \omega_{k, i} t} u_{\mathbf{k}, i}^{r}, \quad v_{\mathbf{k}, i}^{r}(t)=e^{i \omega_{k, i} t} v_{\mathbf{k}, i}^{r}
$$

and $\omega_{k, i}=\sqrt{\mathbf{k}^{2}+m_{i}^{2}}$

The mass eigenstate vacuum is denoted by $|0\rangle_{m}$ : $\alpha_{\mathbf{k}, i}^{r}|0\rangle_{m}=\beta_{\mathbf{k}, i}^{r}|0\rangle_{m}=0$. The anticommutation relations, the wave function orthonormality and completeness relations are the usual ones (cf. Ref. [3]).

The flavor fields are obtained from Eq. (5):

$$
\begin{aligned}
\nu_{\sigma}(x) & =\sum_{r} \int \frac{d^{3} \mathbf{k}}{(2 \pi)^{\frac{3}{2}}}\left[u_{\mathbf{k}, i}^{r}(t) \alpha_{\mathbf{k}, \sigma}^{r}(t)\right. \\
& \left.+v_{-\mathbf{k}, i}^{r}(t) \beta_{-\mathbf{k}, \sigma}^{r \dagger}(t)\right] e^{i \mathbf{k} \cdot \mathbf{x}},
\end{aligned}
$$

with $(\sigma, i)=(e, 1),(\mu, 2)$, with the flavor annihilation operators defined as

$$
\begin{aligned}
\alpha_{\mathbf{k}, \sigma}^{r}(t) & \equiv G_{\theta}^{-1}(t) \alpha_{\mathbf{k}, i}^{r} G_{\theta}(t) \\
\beta_{-\mathbf{k}, \sigma}^{r}(t) & \equiv G_{\theta}^{-1}(t) \beta_{-\mathbf{k}, i}^{r \dagger} G_{\theta}(t) .
\end{aligned}
$$

They annihilate the flavor vacuum $|0(t)\rangle_{f}$ given by

$$
|0(t)\rangle_{f} \equiv G_{\theta}^{-1}(t)|0\rangle_{m}
$$

In the infinite volume limit, the vacuum $|0(t)\rangle_{f}$ for the flavor fields and the vacuum $|0\rangle_{m}$ for the fields with definite masses are unitarily inequivalent vacua $[3,4]$.

One further remark is that the use of the vacuum state $|0\rangle_{m}$ in the computation of the two point Green's functions leads to the violation of the probability conservation [8]. The correct result is instead obtained by the use of the flavor vacuum $|0\rangle_{f}$, which is therefore the relevant vacuum to be used in the computation of the oscillation effects. We will thus use $|0\rangle_{f}$ in our computations in the following.

The explicit expressions for the flavor annihilation/creation operators in the reference frame $\mathbf{k}=(0,0,|\mathbf{k}|)$ are [3]:

$$
\begin{aligned}
\alpha_{\mathbf{k}, e}^{r}(t)= & \cos \theta \alpha_{\mathbf{k}, 1}^{r}+\sin \theta\left(U_{\mathbf{k}}^{*}(t) \alpha_{\mathbf{k}, 2}^{r}\right. \\
& \left.+\epsilon^{r} V_{\mathbf{k}}(t) \beta_{-\mathbf{k}, 2}^{r \dagger}\right) \\
\alpha_{\mathbf{k}, \mu}^{r}(t)= & \cos \theta \alpha_{\mathbf{k}, 2}^{r}-\sin \theta\left(U_{\mathbf{k}}(t) \alpha_{\mathbf{k}, 1}^{r}\right. \\
& \left.-\epsilon^{r} V_{\mathbf{k}}(t) \beta_{-\mathbf{k}, 1}^{r \dagger}\right) \\
\beta_{-\mathbf{k}, e}^{r}(t)= & \cos \theta \beta_{-\mathbf{k}, 1}^{r}+\sin \theta\left(U_{\mathbf{k}}^{*}(t) \beta_{-\mathbf{k}, 2}^{r}\right. \\
& \left.-\epsilon^{r} V_{\mathbf{k}}(t) \alpha_{\mathbf{k}, 2}^{r \dagger}\right) \\
\beta_{-\mathbf{k}, \mu}^{r}(t)= & \cos \theta \beta_{-\mathbf{k}, 2}^{r}-\sin \theta\left(U_{\mathbf{k}}(t) \beta_{-\mathbf{k}, 1}^{r}\right. \\
& \left.+\epsilon^{r} V_{\mathbf{k}}(t) \alpha_{\mathbf{k}, 1}^{r \dagger}\right)
\end{aligned}
$$

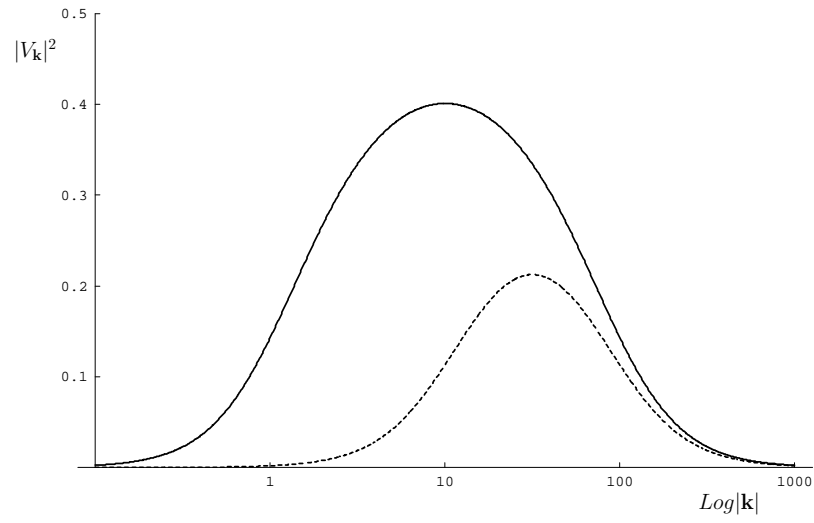

Figure 1. Fermion condensation density $\left|V_{\mathbf{k}}\right|^{2}$ as a function of $|\mathbf{k}|$ and for sample values of the parameters $m_{1}$ and $m_{2}$. Solid line: $m_{1}=1, m_{2}=100$; Long dashed line: $m_{1}=10$, $m_{2}=100$; Short dashed line: $m_{1}=10, m_{2}=1000$.

where $U_{\mathbf{k}}$ and $V_{\mathbf{k}}$ are Bogoliubov coefficients given by:

$$
\begin{aligned}
V_{\mathbf{k}}(t) & =\left|V_{\mathbf{k}}\right| e^{i\left(\omega_{k, 2}+\omega_{k, 1}\right) t}, \\
U_{\mathbf{k}}(t) & =\left|U_{\mathbf{k}}\right| e^{i\left(\omega_{k, 2}-\omega_{k, 1}\right) t}
\end{aligned}
$$

$$
\begin{aligned}
\left|U_{\mathbf{k}}\right| & =\left(\frac{\omega_{k, 1}+m_{1}}{2 \omega_{k, 1}}\right)^{\frac{1}{2}}\left(\frac{\omega_{k, 2}+m_{2}}{2 \omega_{k, 2}}\right)^{\frac{1}{2}} \\
& \times\left(1+\frac{|\mathbf{k}|^{2}}{\left(\omega_{k, 1}+m_{1}\right)\left(\omega_{k, 2}+m_{2}\right)}\right), \\
\left|V_{\mathbf{k}}\right| & =\left(\frac{\omega_{k, 1}+m_{1}}{2 \omega_{k, 1}}\right)^{\frac{1}{2}}\left(\frac{\omega_{k, 2}+m_{2}}{2 \omega_{k, 2}}\right)^{\frac{1}{2}} \\
& \times\left(\frac{|\mathbf{k}|}{\left(\omega_{k, 2}+m_{2}\right)}-\frac{|\mathbf{k}|}{\left(\omega_{k, 1}+m_{1}\right)}\right),
\end{aligned}
$$

with

$$
\left|U_{\mathbf{k}}\right|^{2}+\left|V_{\mathbf{k}}\right|^{2}=1
$$

The function $\left|V_{\mathbf{k}}\right|$ is related to the condensate content of the flavor vacuum [3] as:

$$
{ }_{e, \mu}\left\langle 0\left|\alpha_{\mathbf{k}, i}^{r \dagger} \alpha_{\mathbf{k}, i}^{r}\right| 0\right\rangle_{e, \mu}=\sin ^{2} \theta\left|V_{\mathbf{k}}\right|^{2}, \quad i=1,2
$$

with the same result for antiparticles.

In Fig.1, we plot the fermion condensation density $\left|V_{\mathbf{k}}\right|^{2}$ in function of $|\mathbf{k}|$ and for sample values of the parameters $m_{1}$ and $m_{2} .\left|V_{\mathbf{k}}\right|^{2}$ is zero for $m_{1}=m_{2}$, it has a maximum at $|\mathbf{k}|=\sqrt{m_{1} m_{2}}$ and, for $|\mathbf{k}| \gg \sqrt{m_{1} m_{2}}$, it goes like $\left|V_{\mathbf{k}}\right|^{2} \simeq\left(m_{2}-m_{1}\right)^{2} /\left(4|\mathbf{k}|^{2}\right)$.

From the study of the current algebra, we obtain the flavor charge operators that, in terms of flavor operators, are:

$$
Q_{\sigma}(t)=\sum_{\mathbf{k}, r}\left(\alpha_{\mathbf{k}, \sigma}^{r \dagger}(t) \alpha_{\mathbf{k}, \sigma}^{r}(t)-\beta_{-\mathbf{k}, \sigma}^{r \dagger}(t) \beta_{-\mathbf{k}, \sigma}^{r}(t)\right),
$$


with $\sigma=e, \mu$.

At time $t=0$, the vacuum state is $|0\rangle_{e, \mu}$ and the one electron neutrino state is defined as:

$$
\left|\nu_{e}\right\rangle \equiv \alpha_{\mathbf{k}, e}^{r \dagger}|0\rangle_{e, \mu}
$$

Considering the flavor charge operators, defined as in Eq.(19). We then have (in the Heisenberg representation)

$$
\begin{aligned}
& e, \mu\left\langle 0\left|Q_{e}(t)\right| 0\right\rangle_{e, \mu}=_{e, \mu}\left\langle 0\left|Q_{\mu}(t)\right| 0\right\rangle_{e, \mu}=0 \\
& \mathcal{Q}_{\mathbf{k}, e}^{e}(t)=\left\langle\nu_{e}\left|Q_{e}(t)\right| \nu_{e}\right\rangle \\
& =\left|\left\{\alpha_{\mathbf{k}, e}^{r}(t), \alpha_{\mathbf{k}, e}^{r \dagger}(0)\right\}\right|^{2}+\left|\left\{\beta_{-\mathbf{k}, e}^{r \dagger}(t), \alpha_{\mathbf{k}, e}^{r \dagger}(0)\right\}\right|^{2} \\
& \mathcal{Q}_{\mathbf{k}, \mu}^{e}(t)=\left\langle\nu_{e}\left|Q_{\mu}(t)\right| \nu_{e}\right\rangle \\
& =\left|\left\{\alpha_{\mathbf{k}, \mu}^{r}(t), \alpha_{\mathbf{k}, e}^{r \dagger}(0)\right\}\right|^{2}+\left|\left\{\beta_{-\mathbf{k}, \mu}^{r \dagger}(t), \alpha_{\mathbf{k}, e}^{r \dagger}(0)\right\}\right|^{2}
\end{aligned}
$$

Charge conservation is obviously ensured at any time:

$$
\mathcal{Q}_{\mathbf{k}, e}^{e}(t)+\mathcal{Q}_{\mathbf{k}, \mu}^{e}(t)=1 .
$$

The oscillation formula for the flavor charges are then [8]:

$$
\begin{aligned}
\mathcal{Q}_{\mathbf{k}, e}^{e}(t) & =1-\sin ^{2}(2 \theta)\left[\left|U_{\mathbf{k}}\right|^{2} \sin ^{2}\left(\frac{\omega_{k, 2}-\omega_{k, 1}}{2} t\right)\right. \\
+ & \left.\left|V_{\mathbf{k}}\right|^{2} \sin ^{2}\left(\frac{\omega_{k, 2}+\omega_{k, 1}}{2} t\right)\right], \\
\mathcal{Q}_{\mathbf{k}, \mu}^{e}(t) & =\sin ^{2}(2 \theta)\left[\left|U_{\mathbf{k}}\right|^{2} \sin ^{2}\left(\frac{\omega_{k, 2}-\omega_{k, 1}}{2} t\right)\right. \\
& \left.+\left|V_{\mathbf{k}}\right|^{2} \sin ^{2}\left(\frac{\omega_{k, 2}+\omega_{k, 1}}{2} t\right)\right] .
\end{aligned}
$$

This result is exact. There are two differences with respect to the usual formula for neutrino oscillations: the amplitudes are energy dependent, and there is an additional oscillating term.

For $|\mathbf{k}| \gg \sqrt{m_{1} m_{2}}$ we have $\left|U_{\mathbf{k}}\right|^{2} \longrightarrow 1$ and $\left|V_{\mathbf{k}}\right|^{2} \longrightarrow$ 0 and the traditional formula is recovered.

Similar results are obtained in the case of boson fields [10]. For the $\eta-\eta^{\prime}$ system, the correction may be as large as $20 \%$.

\section{Neutrino mixing contribution to the cosmological constant}

The connection between the vacuum energy density $\left\langle\rho_{v a c}\right\rangle$ and the cosmological constant $\Lambda$ is provided by the well known relation

$$
\left\langle\rho_{v a c}\right\rangle=\frac{\Lambda}{4 \pi G}
$$

where $G$ is the gravitational constant.
The energy-momentum tensor density $\mathcal{T}_{\mu \nu}$ is obtained by varying the action with respect to the metric $g_{\mu \nu}$ :

$$
\mathcal{T}_{\mu \nu}=\frac{2}{\sqrt{-g}} \frac{\delta S}{\delta g^{\mu \nu}(x)},
$$

where the action is

$$
S=\int \sqrt{-g} \mathcal{L}(x) d^{4} x .
$$

In the present case, the energy momentum tensor density is given by

$$
\mathcal{T}_{\mu \nu}(x)=\frac{i}{2}\left(\bar{\Psi}_{m}(x) \gamma_{\mu}(x) \overleftrightarrow{D}_{\nu} \Psi_{m}(x)\right)
$$

where $\overleftrightarrow{D}_{\nu}$ is the covariant derivative:

$D_{\nu}=\partial_{\nu}+\Gamma_{\nu}, \quad \Gamma_{\nu}=\frac{1}{8} \omega_{\nu}^{a b}\left[\gamma_{a}, \gamma_{b}\right], \quad \gamma_{\mu}(x)=\gamma^{c} e_{c \mu}(x)$,

being $\gamma^{c}$ the standard Dirac matrices, and $\bar{\Psi} \overleftrightarrow{D}_{\nu} \Psi=\bar{\Psi} D \Psi-$ $(D \bar{\Psi}) \Psi$. Let us consider the Minkowski metric, we have

$$
\mathcal{T}_{00}(x)=\frac{i}{2}:\left(\bar{\Psi}_{m}(x) \gamma_{0} \overleftrightarrow{\partial}_{0} \Psi_{m}(x)\right):
$$

where : ... : denotes the customary normal ordering with respect to the mass vacuum in the flat space-time.

In terms of the annihilation and creation operators of fields $\nu_{1}$ and $\nu_{2}$, the energy-momentum tensor

$$
T_{00}=\int d^{3} x \mathcal{T}_{00}(x)
$$

is given by

$$
T_{00}^{(i)}=\sum_{r} \int d^{3} \mathbf{k} \omega_{k, i}\left(\alpha_{\mathbf{k}, i}^{r \dagger} \alpha_{\mathbf{k}, i}^{r}+\beta_{-\mathbf{k}, i}^{r \dagger} \beta_{-\mathbf{k}, i}^{r}\right),
$$

with $i=1,2$.

Note that $T_{00}^{(i)}$ is time independent.

The expectation value of $T_{00}^{(i)}$ in the flavor vacuum $|0\rangle_{f}$ gives the contribution $\left\langle\rho_{v a c}^{\text {mix }}\right\rangle$ of the neutrino mixing to the vacuum energy density is:

$$
{ }_{f}\left\langle 0\left|\sum_{i} T_{00}^{(i)}(0)\right| 0\right\rangle_{f}=\left\langle\rho_{v a c}^{m i x}\right\rangle \eta_{00} .
$$

Within the QFT formalism for neutrino mixing, we have

$$
{ }_{f}\left\langle 0\left|T_{00}^{(i)}\right| 0\right\rangle_{f}={ }_{f}\left\langle 0(t)\left|T_{00}^{(i)}\right| 0(t)\right\rangle_{f}
$$

for any t. We then obtain

$$
\begin{aligned}
{ }_{f}\left\langle 0\left|\sum_{i} T_{00}^{(i)}(0)\right| 0\right\rangle_{f} & =\sum_{i, r} \int d^{3} \mathbf{k} \omega_{k, i}\left({ }_{f}\left\langle 0\left|\alpha_{\mathbf{k}, i}^{r \dagger} \alpha_{\mathbf{k}, i}^{r}\right| 0\right\rangle_{f}\right. \\
& \left.+{ }_{f}\left\langle 0\left|\beta_{\mathbf{k}, i}^{r \dagger} \beta_{\mathbf{k}, i}^{r}\right| 0\right\rangle_{f}\right) .
\end{aligned}
$$

By using Eq.(18), we get

$$
\begin{aligned}
{ }_{f}\left\langle 0\left|\sum_{i} T_{00}^{(i)}(0)\right| 0\right\rangle_{f} & =8 \sin ^{2} \theta \int d^{3} \mathbf{k}\left(\omega_{k, 1}+\omega_{k, 2}\right)\left|V_{\mathbf{k}}\right|^{2} \\
& =\left\langle\rho_{v a c}^{\text {mix }}\right\rangle \eta_{00}
\end{aligned}
$$


i.e.

$$
\left\langle\rho_{v a c}^{\operatorname{mix}}\right\rangle=32 \pi^{2} \sin ^{2} \theta \int_{0}^{K} d k k^{2}\left(\omega_{k, 1}+\omega_{k, 2}\right)\left|V_{\mathbf{k}}\right|^{2},
$$

where the cut-off $K$ has been introduced. Eq.(38) is our result: it shows that the cosmological constant gets a non-zero contribution induced from the neutrino mixing [2]. Notice that such a contribution is indeed zero in the no-mixing limit when the mixing angle $\theta=0$ and/or $m_{1}=m_{2}$. Moreover, the contribution is absent in the traditional phenomenological (Pontecorvo) mixing treatment.

We may try to estimate the neutrino mixing contribution by making our choice for the cut-off. If we choose the cut-off proportional to the natural scale appearing in the mixing phenomenon $k_{0} \simeq \sqrt{m_{1} m_{2}}$ [3]: using $K \sim k_{0}$, $m_{1}=7 \times 10^{-3} \mathrm{eV}, m_{2}=5 \times 10^{-2} \mathrm{eV}$, and $\sin ^{2} \theta \simeq 0.3$ [14] in Eq.(38), we obtain

$$
\left\langle\rho_{\text {vac }}^{\text {mix }}\right\rangle=0.43 \times 10^{-47} \mathrm{GeV}^{4}
$$
for $\Lambda$ :

Using Eq.(26), we are in agreement with the upper bound

$$
\Lambda \sim 10^{-56} \mathrm{~cm}^{-2},
$$

Another possible choice is to use the electro-weak scale cutoff: $K \approx 100 \mathrm{GeV}$. We then have

$$
\left\langle\rho_{v a c}^{\operatorname{mix}}\right\rangle=1.5 \times 10^{-15} \mathrm{GeV}^{4}
$$

and

$$
\Lambda \sim 10^{-24} \mathrm{~cm}^{-2},
$$

which is, however, beyond the accepted upper bound.

In a recent paper [15], it was suggested the cut-off scale given by the sum of the two neutrino masses, $K=m_{1}+m_{2}$.

For hierarchical neutrino models, for which $m_{2} \gg m_{1}$, we have, in this case, $K \gg \sqrt{m_{1} m_{2}}$, and thus, if we assume that the modes near the cut-off contribute mainly to the vacuum energy, and take into account the asymptotic properties of $V_{\mathbf{k}}$ :

$$
\left|V_{\mathbf{k}}\right|^{2} \simeq \frac{\left(m_{2}-m_{1}\right)^{2}}{4 k^{2}} \quad k \gg \sqrt{m_{1} m_{2}}
$$

we obtain:

$$
\begin{aligned}
\left\langle\rho_{\text {vac }}^{\text {mix }}\right\rangle & \sim 8 \pi \sin ^{2} \theta\left(m_{2}-m_{1}\right)^{2}\left(m_{2}+m_{1}\right)^{2} \times \\
& \times\left(\sqrt{2}+1+O\left(\frac{m_{1}^{2}}{m_{2}^{2}}\right)\right)
\end{aligned}
$$

and then

$$
\left\langle\rho_{v a c}^{\text {mix }}\right\rangle \propto \sin ^{2} \theta\left(\Delta m^{2}\right)^{2}
$$

in the limit $m_{2} \gg m_{1}$.

In Ref.[15], the corresponding $\Delta m^{2}$ is given by the solar neutrino data: $\Delta m^{2} \simeq 10^{-5} \mathrm{eV}^{2}$, resulting in a contribution of the right order.

\section{Quantum fields and mixing in ex- panding universe}

In this Section we present a preliminary study of mixed fields on a time-dependent gravitational background. For simplicity, we consider the case of neutral scalar fields, the case of fermionic fields and neutrino oscillations will be considered elsewhere.

\subsection{Free fields in expanding universe}

We start by quantizing a free neutral scalar field $\phi$ in the Friedmann-Robertson-Walker (FRW) space-time with flat spatial sections, characterized by metrics of the form

$$
d s^{2}=g_{\mu \nu} d x^{\mu} d x^{\nu}=d t^{2}-a^{2}(t) d \mathbf{x}^{2},
$$

where $a(t)$ is the scale factor.

A flat FRW space-time is a conformally flat space-time. Indeed, by replacing the coordinate $t$ by the conformal time $\eta$,

$$
\eta(t)=\int_{t_{0}}^{t} \frac{d t}{a(t)},
$$

where $t_{0}$ is an arbitrary constant, the line element takes the form

$$
d s^{2}=a^{2}(\eta)\left[d \eta^{2}-d \mathbf{x}^{2}\right],
$$

where $a(\eta)$ is the scale factor expressed through the new variable $\eta$. Introducing the auxiliary field $\chi=a(\eta) \phi$, it is possible to show that the evolution of a scalar field $\phi$ in a flat FRW metric is mathematically equivalent to the dynamics of the auxiliary field $\chi$ in the Minkowski metric [16]. The information about the influence of gravitational field on $\phi$ is contained in the time-dependent mass $m_{e f f}(\eta)$ defined by

$$
m_{e f f}^{2}(\eta)=m^{2} a^{2}-\frac{a^{\prime \prime}}{a},
$$

where the prime ' denotes the derivative with respect to $\eta$.

The field $\chi$ can be quantized in the standard fashion by introducing the equal time commutation relations

$$
[\chi(\mathbf{x}, \eta), \pi(\mathbf{y}, \eta)]=i \delta^{3}(\mathbf{x}-\mathbf{y}),
$$

where $\pi=\chi^{\prime}$ is the canonical momentum.

The Hamiltonian of the quantum field $\chi$ is

$$
H(\eta)=\frac{1}{2} \int d^{3} \mathbf{x}\left[\pi^{2}+(\nabla \chi)^{2}+m_{\text {eff }}^{2}(\eta) \chi^{2}\right] .
$$

Note that the energy of the field $\chi$ is not conserved; this leads to the possibility of particle creation in the vacuum. The energy for new particles is supplied by gravitational field.

The field $\chi$ is expanded as

$$
\chi(\mathbf{x}, \eta)=\int \frac{d^{3} \mathbf{k}}{(2 \pi)^{\frac{3}{2}}} \frac{e^{i \mathbf{k x}}}{\sqrt{2}}\left(v_{k}^{*}(\eta) a_{\mathbf{k}}+v_{k}(\eta) a_{-\mathbf{k}}^{\dagger}\right),
$$


where the mode functions $v_{k}(\eta)$ obey the equations

$$
v_{k}^{\prime \prime}+\omega_{k}^{2}(\eta) v_{k}=0, \quad \omega_{k}(\eta)=\sqrt{k^{2}+m_{e f f}^{2}(\eta)}
$$

and satisfy the following normalization condition

$$
v_{k}^{\prime} v_{k}^{*}-v_{k} v_{k}^{\prime *}=2 i
$$

We have $v_{k}(\eta)=v_{-k}(\eta)$ as follows from the relation $\left(\chi_{\mathbf{k}}\right)^{*}=\chi_{-\mathbf{k}}$ and $\left(a_{\mathbf{k}}\right)^{*}=a_{\mathbf{k}}^{\dagger}$, where

$$
\chi_{\mathbf{k}}(\eta)=\frac{1}{\sqrt{2}}\left(v_{k}^{*}(\eta) a_{\mathbf{k}}+v_{k}(\eta) a_{-\mathbf{k}}^{\dagger}\right)
$$

The annihilation operators are given in terms of $\chi_{\mathbf{k}}(\eta)$ and $v_{k}(\eta)$ by:

$$
a_{\mathbf{k}}=\sqrt{2} \frac{v_{k}^{\prime} \chi_{\mathbf{k}}-v_{k} \chi_{\mathbf{k}}^{\prime}}{v_{k}^{\prime} v_{k}^{*}-v_{k} v_{k}^{\prime *}}
$$

Note that $a_{\mathbf{k}}$ is time-independent; the commutation relations are:

$$
\left[a_{\mathbf{k}}, a_{\mathbf{k}^{\prime}}^{\dagger}\right]=\delta^{3}\left(\mathbf{k}-\mathbf{k}^{\prime}\right), \quad\left[a_{\mathbf{k}}, a_{\mathbf{k}^{\prime}}\right]=\left[a_{\mathbf{k}}^{\dagger}, a_{\mathbf{k}^{\prime}}^{\dagger}\right]=0 .
$$

The vacuum state annihilated by $a_{\mathbf{k}}$ is denoted by $|0(\eta)\rangle$ : $a_{\mathbf{k}}|0(\eta)\rangle=0$.

\subsection{The instantaneous vacuum}

The Hamiltonian Eq.(51) is time dependent, then we can define an "instantaneous" vacuum by selecting an arbitrary time $\eta_{0}$ and defining the vacuum $\left|0\left(\eta_{0}\right)\right\rangle$ as the lowest energy eigenstate of the Hamiltonian $H\left(\eta_{0}\right)$ computed at the time $\eta_{0}$.

The mode functions $v_{k}\left(\eta_{0}\right)$, corresponding to the vacuum $\left|0\left(\eta_{0}\right)\right\rangle$, are obtained by computing the expectation value $\left\langle 0(\eta)\left|H\left(\eta_{0}\right)\right| 0(\eta)\right\rangle$ in the vacuum state $|0(\eta)\rangle$ determined by arbitrary chosen mode functions $v_{k}(\eta)$ and then minimizing that expectation value with respect to all possible choice of $v_{k}(\eta)[16]$.

The Hamiltonian Eq.(51) expressed through the annihilations operators $a_{\mathbf{k}}$ defined by the arbitrary mode functions $v_{k}(\eta)$ is

$$
\begin{aligned}
H(\eta) & =\frac{1}{4} \int d^{3} \mathbf{k}\left[\left(v_{k}^{\prime 2}(\eta)+\omega_{k}^{2}(\eta) v_{k}^{2}(\eta)\right)^{*} a_{\mathbf{k}} a_{-\mathbf{k}}\right. \\
& +\left(v_{k}^{\prime 2}(\eta)+\omega_{k}^{2}(\eta) v_{k}^{2}(\eta)\right) a_{\mathbf{k}}^{\dagger} a_{-\mathbf{k}}^{\dagger} \\
& \left.+\left(\left|v_{k}^{\prime}(\eta)\right|^{2}+\omega_{k}^{2}(\eta)\left|v_{k}(\eta)\right|^{2}\right)\left(2 a_{\mathbf{k}}^{\dagger} a_{\mathbf{k}}+\delta^{3}(0)\right)\right]
\end{aligned}
$$

Since $a_{\mathbf{k}}|0(\eta)\rangle=0$, we have

$$
\begin{aligned}
\left\langle 0(\eta)\left|H\left(\eta_{0}\right)\right| 0(\eta)\right\rangle & =\frac{1}{4} \delta^{3}(0) \int d^{3} \mathbf{k}\left(\left|v_{k}^{\prime}(\eta)\right|^{2}\right. \\
& \left.+\omega_{k}^{2}(\eta)\left|v_{k}(\eta)\right|^{2}\right)_{\eta=\eta_{0}}
\end{aligned}
$$

and the density energy is

$$
\rho=\frac{1}{4} \int d^{3} \mathbf{k}\left(\left|v_{k}^{\prime}(\eta)\right|^{2}+\omega_{k}^{2}(\eta)\left|v_{k}(\eta)\right|^{2}\right)_{\eta=\eta_{0}} .
$$

At fixed value of the momentum $k$, if $\omega_{k}^{2}\left(\eta_{0}\right)>0$, it is possible to show that the mode functions $v_{k}(\eta)$ that minimize $\rho$ satisfy the following initial conditions at $\eta=\eta_{0}[16]$ :

$$
v_{k}\left(\eta_{0}\right)=\frac{1}{\sqrt{\omega_{k}\left(\eta_{0}\right)}}, \quad v_{k}^{\prime}\left(\eta_{0}\right)=i \sqrt{\omega_{k}\left(\eta_{0}\right)} .
$$

The mode functions satisfying the Eq.(60) define the annihilation operators $a_{\mathbf{k}}^{0}$ of the vacuum $\left|0\left(\eta_{0}\right)\right\rangle: a_{\mathbf{k}}^{0}\left|0\left(\eta_{0}\right)\right\rangle=0$ through which the Hamiltonian $H\left(\eta_{0}\right)$ at time $\eta_{0}$ is expressed as

$$
H\left(\eta_{0}\right)=\int d^{3} \mathbf{k} \omega_{k}\left(\eta_{0}\right)\left(a_{\mathbf{k}}^{0 \dagger} a_{\mathbf{k}}^{0}+\frac{1}{2} \delta^{3}(0)\right) .
$$

The zero point energy density of quantum field in the vacuum state $\left|0\left(\eta_{0}\right)\right\rangle$ is

$$
\rho_{0}=\frac{1}{2} \int d^{3} \mathbf{k} \omega_{k}\left(\eta_{0}\right)
$$

This quantity is time dependent, but, considering the problem of particle oscillations in the present time, since the time scale of mixing phenomena are much smaller than the cosmological time scale, we can neglect the particle creation in the vacuum due to the gravitational field and we may consider $\rho \simeq$ constant at the present time and then we renormalize in usual way.

For further analysis of the vacuum structure in a curved background see refs.[17].

\subsection{Mixed fields in expanding universe}

The boson mixing relations in FRW space-time are generalized as

$$
\begin{aligned}
& \chi_{A}(\mathbf{x}, \eta)=\chi_{1}(\mathbf{x}, \eta) \cos \theta+\chi_{2}(\mathbf{x}, \eta) \sin \theta \\
& \chi_{B}(\mathbf{x}, \eta)=-\chi_{1}(\mathbf{x}, \eta) \sin \theta+\chi_{2}(\mathbf{x}, \eta) \cos \theta(63)
\end{aligned}
$$

We now proceed in a similar way to what has been done in Ref.[10] for bosons in flat space-time and recast Eqs.(63) into the form:

$$
\begin{aligned}
& \chi_{A}(\mathbf{x}, \eta)=G_{\theta}^{-1}(\eta) \chi_{1}(\mathbf{x}, \eta) G_{\theta}(\eta) \\
& \chi_{B}(\mathbf{x}, \eta)=G_{\theta}^{-1}(\eta) \chi_{2}(\mathbf{x}, \eta) G_{\theta}(\eta)
\end{aligned}
$$

and similar ones for $\pi_{A}(\mathbf{x}, \eta), \pi_{B}(\mathbf{x}, \eta) . G_{\theta}(\eta)$ denotes the operator which implements the mixing transformations (63):

$$
G_{\theta}(\eta)=\exp [\theta S(\eta)]
$$

with

$$
\begin{aligned}
S(\eta) & =-i \int d^{3} \mathbf{x}\left(\pi_{1}(\mathbf{x}, \eta) \chi_{2}(\mathbf{x}, \eta)\right. \\
& \left.-\pi_{2}(\mathbf{x}, \eta) \chi_{1}(\mathbf{x}, \eta)\right)
\end{aligned}
$$


We have, explicitly

$$
\begin{aligned}
S(\eta) & =\int d^{3} \mathbf{k}\left(U_{\mathbf{k}}^{*}(\eta) a_{\mathbf{k}, 1}^{\dagger} a_{\mathbf{k}, 2}-V_{\mathbf{k}}^{*}(\eta) a_{-\mathbf{k}, 1} a_{\mathbf{k}, 2}\right. \\
& \left.+V_{\mathbf{k}}(\eta) a_{-\mathbf{k}, 1}^{\dagger} a_{\mathbf{k}, 2}^{\dagger}-U_{\mathbf{k}}(\eta) a_{\mathbf{k}, 1} a_{\mathbf{k}, 2}^{\dagger}\right)
\end{aligned}
$$

where $U_{\mathbf{k}}(\eta)$ and $V_{\mathbf{k}}(\eta)$ are Bogoliubov coefficients given by

$$
\begin{aligned}
U_{\mathbf{k}}(\eta) & \equiv i\left[v_{k, 1}^{*}(\eta) v_{k, 2}(\eta)-v_{k, 2}^{\prime}(\eta) v_{k, 1}^{*}(\eta)\right] \\
V_{\mathbf{k}}(\eta) & \equiv-i\left[v_{k, 1}^{\prime}(\eta) v_{k, 2}(\eta)-v_{k, 2}^{\prime}(\eta) v_{k, 1}(\eta)\right]
\end{aligned}
$$

and satisfy the relation

$$
\left|U_{\mathbf{k}}\right|^{2}-\left|V_{\mathbf{k}}\right|^{2}=1 .
$$

Similar results can be obtained in the case of fermion mixing.

\subsection{The de Sitter space-time}

The de Sitter space-time is characterized by a scale factor of the form

$$
a(t) \sim e^{H t},
$$

where $H=\dot{a} / a>0$ is the Hubble constant. The conformal time $\eta$, the scale factor $a(\eta)$ and the effective frequency are given by

$$
\begin{array}{r}
\eta=-\frac{1}{H} e^{-H t}, \quad a(\eta)=-\frac{1}{H \eta} \\
\omega_{k}^{2}(\eta)=k^{2}+\left(\frac{m^{2}}{H^{2}}-2\right) \frac{1}{\eta^{2}} .
\end{array}
$$

The conformal time $\eta$ ranges from $-\infty$ to 0 when the proper time $t$ goes from $-\infty$ to $\infty$. The origin of $\eta$ is chosen so that the infinite future corresponds to $\eta=0$.

For this space-time, the general solution of Eq.(53) is

$$
v_{k}(\eta)=\sqrt{k|\eta|}\left[A J_{n}(k|\eta|)+B Y_{n}(k|\eta|)\right],
$$

with $n=\sqrt{\frac{9}{4}-\frac{m^{2}}{H^{2}}}, \mathrm{~A}$ and B constants, and $J_{n}(k|\eta|)$ and $Y_{n}(k|\eta|)$ Bessel functions.

In the de Sitter space-time, a suitable vacuum state is the Bunch-Davies vacuum, defined as the Minkowski vacuum in the early time limit $(\eta \rightarrow-\infty)$ :

$$
v_{k}(\eta)=\frac{1}{\sqrt{\omega_{k}}} e^{i \omega_{k} \eta}, \quad \eta \rightarrow-\infty .
$$

In this case, the mode functions are

$$
v_{k}(\eta)=\sqrt{\frac{\pi|\eta|}{2}}\left[J_{n}(k|\eta|)-i Y_{n}(k|\eta|)\right],
$$

with $n=\sqrt{\frac{9}{4}-\frac{m^{2}}{H^{2}}}$.

Assuming for the present time, i.e. the actual time relative to the observer,

$$
t=0 \Rightarrow \eta=-\frac{1}{H} \Rightarrow a(\eta)=1
$$

the mixing transformations in the flat space-time are good approximation of those in FRW space-time Eqs.(63), since the time scale of mixing phenomena are much smaller than the cosmological time scale. The results obtained in Section III describe the contribution to the value of the cosmological constant given by the neutrino mixing at present time.

\section{Conclusions}

The neutrino mixing is a possible source for the cosmological constant. Indeed, the non-perturbative vacuum structure associated with neutrino mixing leads to a non-zero contribution to the value of the cosmological constant [2]. The value of $\Lambda$ consistent with its accepted upper bound is found by using the natural scale of the neutrino mixing cut-off. The origin of the contribution here discussed is completely different from that of the ordinary contribution to the vacuum zero energy of a massive spinor field: it is the mixing phenomenon which provides the vacuum energy contribution discussed in this paper.

\section{Acknowledgments}

We thank MURST, INFN, INFM and ESF Program COSLAB for financial support.

\section{References}

[1] Ya. B. Zeldovich, I. D. Novikov, Structure and evolution of the universe (Izdatel'stvo Nauka, Moscow, 1975); V. Sahni and A. A. Starobinsky, Int. J. Mod. Phys. D 9, 373 (2000).

[2] M. Blasone, A. Capolupo, S. Capozziello, S. Carloni, and G. Vitiello, Phys. Lett. A 323, 182 (2004); [hep-th/0410196].

[3] M. Blasone and G. Vitiello, Annals Phys. 244, 283 (1995) [Erratum-ibid. 249, 363 (1995)].

[4] K. C. Hannabuss and D. C. Latimer, J. Phys. A 33, 1369 (2000); J. Phys. A 36, L69 (2003).

[5] M. Binger and C. R. Ji, Phys. Rev. D 60, 056005 (1999); C. R. Ji and Y. Mishchenko, Phys. Rev. D 64, 076004 (2001); Phys. Rev. D 65, 096015 (2002).

[6] K. Fujii, C. Habe, and T. Yabuki, Phys. Rev. D 59, 113003 (1999) [Erratum-ibid. D 60 (1999) 099903]; Phys. Rev. D 64, 013011 (2001); K. Fujii, C. Habe, and M. Blasone, [hep$\mathrm{ph} / 0212076]$.

[7] M. Blasone and G. Vitiello, Phys. Rev. D 60, 111302 (1999); M. Blasone, P. Jizba, and G. Vitiello, Phys. Lett. B 517, 471 (2001);

[8] M. Blasone, P. A. Henning, and G. Vitiello, Phys. Lett. B 451, 140 (1999); M. Blasone, P. P. Pacheco, and H. W. C. Tseung, Phys. Rev. D 67, 073011 (2003).

[9] M. Blasone, J. Magueijo, and P. Pires-Pacheco, [hep$\mathrm{ph} / 0307205]$.

[10] M. Blasone, A. Capolupo, O. Romei, and G. Vitiello, Phys. Rev. D 63, 125015 (2001); A. Capolupo, C. R. Ji, Y. Mishchenko, and G. Vitiello, Phys. Lett. B 594, 135 (2004). 
[11] M. Blasone, A. Capolupo, and G. Vitiello, Phys. Rev. D 66, 025033 (2002).

[12] M. Blasone and J. Palmer, Phys. Rev. D 69, 057301 (2004).

[13] A. Capolupo, Ph.D. thesis (2004), [hep-th 0408228];

[14] G.L. Fogli, E. Lisi, A. Marrone, A. Melchiorri, A. Palazzo, P. Serra, and J. Silk, [hep-ph/0408045].
[15] G. Barenboim and N. E. Mavromatos, [hep-ph/0406035]; N. E. Mavromatos, [gr-qc/0411067].

[16] V. F. Mukhanov, S. Winitzki, Introduction to Quantum Fields in Classical Backgrounds, Lecture notes - 2004

[17] M. Martellini, P. Sodano, and G. Vitiello, Nuovo Cim. A 48, 341 (1978); E. Alfinito, R. Manka, and G. Vitiello, Class. Quant. Grav. 17, 93 (2000). 